\title{
Percepción sobre los obstáculos cotidianos para la superación en la vida de las mujeres del Quindío*
}

\author{
Irina Artamonova**, Xatlí Murillo***, José Daniel Mosquera****
}

\section{Resumen}

Introducción. En el siguiente artículo se presentan los resultados de un estudio de la situación de las mujeres en el departamento del Quindío-Colombia en sus contextos laboral, de hogar y de educación. En este trabajo se estudiaron las correlaciones existentes entre los factores que definen la percepción de vida de estas mujeres, su satisfacción personal y los obstáculos en su vida cotidiana. Objetivos. Ha sido el estudio de la situación de las mujeres en el departamento del Quindío en su contexto laboral, de hogar y de salud. Métodos. Se encuestaron 399 mujeres del departamento del Quindío, escogidas de forma aleatoria, y se les aplicó directamente un instrumento con preguntas cualitativas; luego, con base en la definición de las variables de interés, se procedió al análisis cuantitativo. Resultados. Se destacan problemas laborales, económicos, culturales relacionados con tendencias machistas de la sociedad. Les siguen problemas relacionadas con la educación, el maltrato, el cuidado de los hijos, el hogar, la discriminación y la actual cultura política. Conclusiones. Existe evidente relación entre la situación laboral, la pobreza, la desigualdad de ingresos, las altas cifras de maltrato y violencia, y la discriminación contra el género femenino en la región del Quindío.

Palabras clave: estudios de género, problemática de las mujeres, desigualdad, violencia, discriminación.

\section{Perception about daily life obstacles for life improvement among women from Quindío}

\section{Abstract}

Introduction. This article presents the results of a study about the situation of women in the Quindío province, Colombia, within the labor, home and education contexts. The research work approaches the existing correlations between the factors that define the perception of life these women have, their personal satisfaction and the obstacles they face in their daily lives. Objectives. Study the situation of women from Quindío within the labor, home and education contexts. Methods. 399 women from Quindío were surveyed. The sample was made at random and an instrument with qualitative questions was applied. Then, based on the definition of the variables of interest, the quantitative analysis was performed. Results. Labor, economic and cultural problems related to the sexist tendencies of the society are remarked. Then, there are problems related to education, abuse, children care, home, discrimination and the current political culture. Conclusions. There is an evident relationship between the labor situation, the poverty, the inequality of income, the high abuse and violence figures and the discrimination against women in Quindío.

Key words: gender studies, women problems, inequality, violence, discrimination.

\section{Percepção sobre os obstáculos cotidianos para a superação na vida das mulheres do Quindío}

\section{Resumo}

Introdução. No seguinte artigo se apresentam os resultados de um estudo da situação das mulheres no departamento do Quindío-Colômbia em seus contextos trabalhista, de lar e de educação. Neste trabalho se estudaram as correlações existentes

\footnotetext{
* $\quad$ El proyecto que dio como resultado este artículo "Percepción sobre los obstáculos cotidianos para la superación en la vida de las mujeres del Quindío" Correo de contacto: iartamonova@uniquindio.edu.co

** Universidad del Quindío, (Colombia). Doctorado (c) en Física Educativa, Maestría en Enseñanza de las Matemáticas, Magister de Ciencias Físicas y Matemáticas, Física

*** Universidad del Quindío, (Colombia). Comunicadora social y periodista de la Universidad Externado de Colombia; Magister en Estudios de Género, Área Mujer y Desarrollo.

**** Universidad Autónoma de Nuevo León (México). Ingeniero industrial, Magister en Ingeniería de Calidad. Magister (c) en Ingeniería de Sistemas.
}

Autor para correspondencia: Irina Artamonova, e-mail: iartamonova@uniquindio.edu.co Artículo recibido: 28/08/2013; Artículo aprobado: 15/05/2015. 
entre os fatores que definem a percepção de vida destas mulheres, sua satisfação pessoal e os obstáculos em sua vida cotidiana. Objetivos. Foi o estudo da situação das mulheres no departamento do Quindío em seu contexto trabalhista, de lar e de saúde. Métodos. Se entrevistaram 399 mulheres do departamento do Quindío, escolhidas de forma aleatória, e se lhes aplicou diretamente um instrumento com perguntas qualitativas; depois, com base na definição das variáveis de interesse, procedeu-se à análise quantitativa. Resultados. Destacam-se problemas trabalhistas, econômicos, culturais relacionados com tendências machistas da sociedade. Seguem problemas relacionadas com a educação, o maltrato, o cuidado dos filhos, o lar, a discriminação e a atual cultura política. Conclusões. Existe evidente relação entre a situação trabalhista, a pobreza, a desigualdade de rendimentos, as altas cifras de maltrato e violência, e a discriminação contra o gênero feminino na região do Quindío.

Palavras Chaves: estudos de gênero, problemática das mulheres, desigualdade, violência, discriminação.

\section{Introducción}

Colombia ocupa el puesto 55 entre 200 países en desigualdad de género en el mundo, de acuerdo con el Global Gender Gap Report (Hausmann, Tyson y Zadich, 2010, 106). Sin embargo, a la población en general y especialmente a aquellos que están encargados de elaborar las políticas de desarrollo del país, este tema parece de poca importancia y posiblemente debido a la larga tradición de familias patriarcales, donde la mujer ha jugado fundamentalmente un papel de ama de casa (Solano y Flórez, 2013, 16-28), la sociedad considera que la situación actual de la mujer es normal. Solo un estudio directo sobre la situación personal de las mujeres en cada región del país puede dar herramientas válidas para valorar el estado de igualdad de género (Monge, 2006, 40-50) y la vulnerabilidad del sector femenino en la sociedad, y de esta manera poder elaborar políticas específicas tendentes a mejorar la situación del llamado sexo débil de la sociedad (Gaviria, y Henao, 2001, 23-38).

\section{Breve caracterización de la pobla- ción del estudio}

Según las proyecciones del DANE basadas en el censo del año 2005, para el año 2010 la población el departamento del Quindío era de 550 mil habitantes, equivalente a $1,21 \%$ de la población nacional. Para el departamento del Quindío, el 50,9 \% son mujeres (DANE, 2005). En el 2009 , el 30,86 \% de la población ocupada quindiana estaba laborando en el comercio y
$22,93 \%$ en el área de los servicios, $15,65 \%$ en agricultura, $8,82 \%$ en la industria, $7,81 \%$ en actividad inmobiliaria, $4,82 \%$ en la construcción y $8,17 \%$ en transporte y comunicaciones (Gómez, 2010).

El objetivo central de este trabajo ha sido el estudio de la situación de las mujeres en el departamento del Quindío en su contexto laboral, de hogar y de salud, buscando su correlación existente entre factores que definen la percepción de la vida de las mujeres quindianas usando técnicas de estadística multivariada.

\section{Métodos}

Para la realización del estudio de campo conducente a determinar los obstáculos con que se encuentran en su vida las mujeres quindianas, se utilizó la técnica de muestreo aleatorio simple. Se les dio a las mujeres la oportunidad de definir por sí mismas los obstáculos o dificultades que tienen en su vida cotidiana.

Para la determinación de la muestra se tuvo en cuenta la probabilidad $p$ del $50 \%(p=0.5)$ para cualquiera de las dificultades en la vida de las mujeres que pudieran presentar, obteniendo la muestra con mayor tamaño posible. Con los pronósticos poblacionales hechos por DANE después del censo de 2005 se esperaba para el año 2010 que en el Quindío residieran N = 215.848 mujeres con edad de 14 o más años. Finalmente se empleó la ecuación (1) para el cálculo de la muestra de una proporción para una población finita (Martínez, 2005, 392-393): 


$$
n=Z_{\alpha}^{2} \frac{N * p * q}{i^{2} *(N-1)+Z_{\alpha}^{2} p * q}
$$

(ecuación 1)

Donde $Z_{\alpha}$ es el valor correspondiente a la distribución normal estándar (siendo a el nivel de confianza elegido). Se eligió un nivel de confianza de $95 \%$ para el cual $\alpha=0,05$ y $Z_{\alpha}=1,96$, tanto $p$ como $q$ de 0,5 y un error de estimación del $5 \%(i=0,05)$. Con estos datos se pudo estimar el tamaño de la muestra poblacional igual a 399 encuestas.

Las encuestas fueron aplicadas en la primera semana de diciembre de 2010 por 89 mujeres participantes del diplomado "Reinventando la ciudadanía de las mujeres" (Crónica del Quindío, 2010) capacitadas por académicos en la Universidad del Quindío. De tal manera se logró aplicar la encuesta en lugares diferentes, haciendo énfasis en no encuestar a mujeres menores de 14 años, dado que mujeres menores a este límite de edad pueden presentar dificultades en el entendimiento del instrumento.

\section{Resultados}

Entre las mujeres encuestadas se encontraban: amas de casa, madres comunitarias, estudiantes, secretarias, empleadas domésticas, comerciantes, así como mujeres con alto nivel de estudios universitarios: médicas, enfermeras, psicólogas, abogadas, maestras, administradoras e ingenieras. Entre las mujeres encuestadas, el $41,9 \%$ eran solteras, casadas $28,3 \%$, en unión libre estaban $10,8 \%$ de las mujeres, $10,0 \%$ eran separadas y $5,8 \%$, viudas. La gran mayoría de las mujeres tenían hijos y solo un $23 \%$ de mujeres no tenían hijos al momento de aplicar la encuesta.

\section{Dificultades generales en la vida de las mujeres}

Todas las dificultades en la vida cotidiana de las mujeres quindianas se agruparon en 7 categorías: económicas, laborales, culturales, de educación, maltrato, cuidado de hijos, hogar, cultura política y de discriminación (Figura 1).

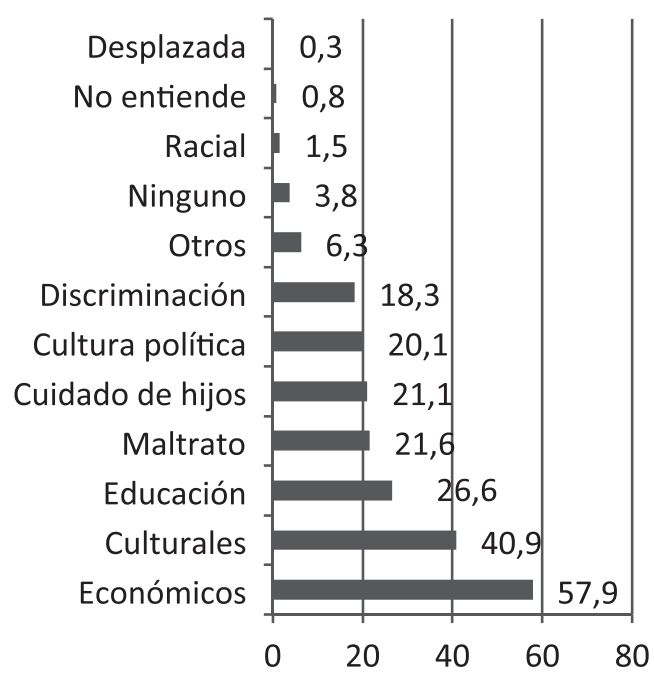

Figura 1. Obstaculos o dificultades en la vida de las mujeres, Quindío, 2010

\section{Problemas económicos, laborales}

La mayoria de las mujeres se encontrarón con problemas de tipo económico o laboral $(57,9$ $\%)$. De la poblacion, solo el $38,1 \%$ tenía trabajo formal y el $8,5 \%$ se ocupaba en oficios varios o trabajaba en forma independiente como vendedoras por catálogo o empleadas domésticas. El $17,5 \%$ de las mujeres se identificó como "amas de casa". La tasa de ocupación calculada fue de $46,6 \%$ para las mujeres quindianas (Figura 2). 


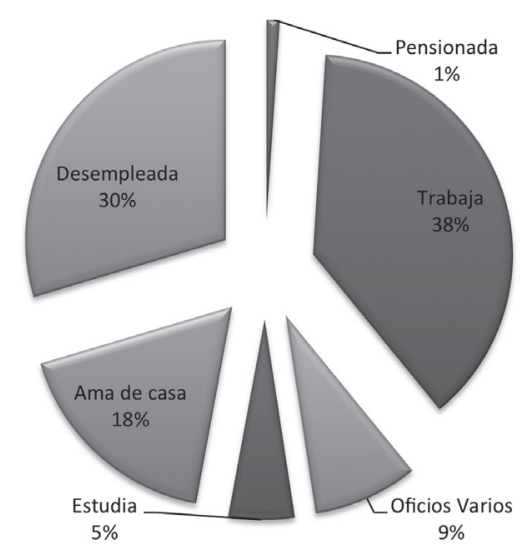

Figura 2. Ocupaciones de las mujeres en el Quindío para 2010

Dicha tasa está por encima del 43,7\% que es la tasa de ocupación para las mujeres en el ámbito nacional, registrada por el DANE para el año 2010 Departamento Administrativo Nacional de Estadística (DANE, 2011). Para el mismo año la tasa de ocupación para los hombres fue de $67,5 \%$ a escala nacional. En la investigación, el 31,6\% de la poblacion mencionó como mayor obstáculo en su vida la falta de empleo y oportunidades en la región.

En el periodo 2001-2010 la diferencia entre las tasas de ocupación entre los hombres y las mujeres en el país está variando entre 23,8 y $26,7 \%$. El nivel de desempleo o desocupación de las mujeres quindianas fue de $29,6 \%$ y es mucho más alto en comparación con el nivel de desempleo $(15,6 \%)$ promedio nacional para el año 2010, calculado por el DANE. La diferencia en el nivel de desempleo entre hombres y mujeres en el nivel nacional varía entre 5,9\% y 7,5\% para los últimos 10 años. Cabe resaltar que en las mejores temporadas la tasa de desempleo más baja para las mujeres se ubicaba en $14,6 \%$ en el año 2007 a escala nacional.

El $15,3 \%$ de las mujeres está luchando con la pobreza y dependencia económica. Entre las mujeres estudiadas solo $1,1 \%$ vivían de las pensiones, pero de ellas ninguna de las mujeres de 65 años o más tenía una pension (Figura 3).

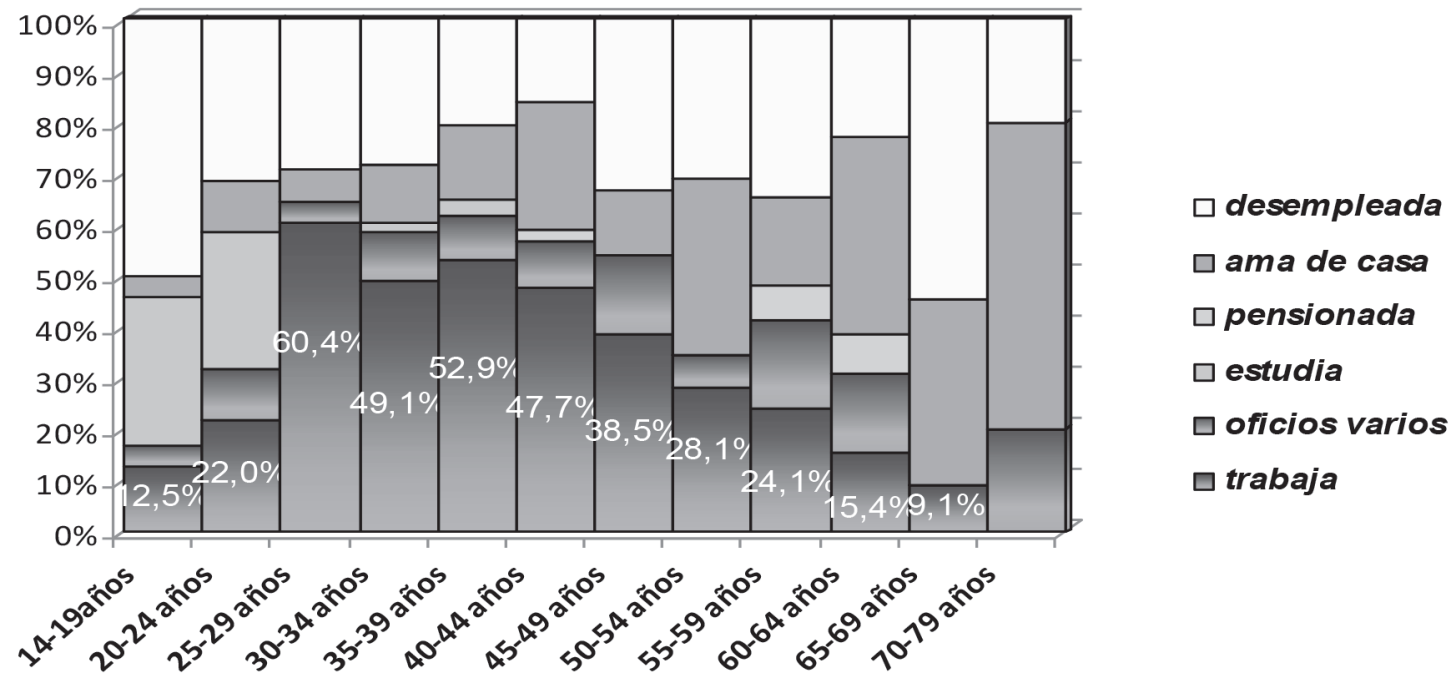

Figura 3. Ocupación de las mujeres por rangos de edad 
La tercera categoría más mencionada, con un $12,8 \%$ de las mujeres, es la discriminación por edad respecto al trabajo. El 10,0 \% de las mujeres ha mencionado casos de discriminación de género con relación al trabajo. Las expresiones "Muchas de las veces por ser mujer, no obtienen buen trabajo; por ser mujeres nos quitan muchos trabajos" caracterizan las situaciones de desigualdad cuando se eligen hombres en vez de mujeres para ser contratados en un empleo.

El 8,0 \% de mujeres mencionan haber sentido la discriminación salarial. Dicha discriminación ha constituido un tema ampliamente estudiado: Amnistía Internacional. (2004), Portafolio. co (2010), El Colombiano. (2012), Rodríguez P. G. y Flórez V. G. (2007, 87-108), Cano, C. y Orozco, M. (2011, 59-97), Daza-Caicedo, $S$ y Pérez-Bustos, T. (2008, 59-97), Buquet Corleto, A.; Cooper, J. y Rodríguez, H. (2010, 20, 29-31, 34, 55), Gutiérrez, A. (2010, 47-69), Del Castillo, M; Roth D. A. N.; Patiño W. C. I; Higuiera, R. R. y Chacón N. B. O. A. (2008, 217255) por economistas y otros investigadores de las ciencias sociales. Se han encontrado con la discriminación por la maternidad 2,3\% de mujeres: "a veces los trabajos no se los dan a las mujeres por tener hijos y ser madres solteras o por estar embarazada" (Fuente propia: encuestas a las mujeres). En Europa la tasa de empleo es del $75,4 \%$ para mujeres sin hijos, y del $61,1 \%$ para las mujeres con hijos. Los resultados de esta investigación hacen referencia a que entre las mujeres quindianas de 24-29 años tienen empleo formal o no formal $69,7 \%$ de las mujeres sin hijos y solamente $45,8 \%$ de las mujeres con hijos.

\section{Problemas culturales}

La mayoría de las sociedades actuales presentan como rasgo estructural el dominio masculino. Históricamente, en muchas sociedades patriarcales, las mujeres han sido vistas como el "sexo débil". La estructura social, con su claro dominio de lo masculino sobre lo femenino, determina en gran medida las relaciones entre sexos.

Dos fenómenos bien diferenciados ilustran con claridad esta situación: el sexismo que se ejerce contra las mujeres, y el "enmudecimiento" de estas. El sexismo tradicional se basa en una supuesta inferioridad de las mujeres como grupo. En las actitudes hacia las mujeres, la manifestación ideológica del patriarcado es el paternalismo, la justificación de la dominancia masculina. El componente hostil de esta ideología es el "paternalismo dominante", la creencia de que los hombres deberían tener más poder que las mujeres y el correspondiente temor de que las mujeres podrían usurpar el poder de los hombres. Esta actitud aparece en el dominio público, por ejemplo, cuando las mujeres experimentan más discriminación en el trabajo.

El mundo de las mujeres, hasta hace apenas un siglo, se reducía al hogar, y su papel en la sociedad se limitaba a ser buena hija, buena madre y buena esposa. El mundo, fuera de las paredes de la casa, no era para ellas, no les estaba permitido, sino que lo vivían a través de la ventana de su casa. Eran las eternas espectadoras, nunca la primera actriz.

Antes los terratenientes tenían sustento en su casa y la mujer podía trabajar en su propio hogar. Con el crecimiento de la población y el apoderamiento de las tierras por un grupo minoritario, la única opción de conseguir sustento es el empleo formal o no formal. Sin embargo, aunque la mujer tiene la necesidad de ser una trabajadora, los estereotipos de la sociedad aún no se han cambiado, y la siguen viendo como frágil e incapaz, es decir, con la carencia de instrumentos y preparación, actitudes que mantienen a las mujeres alejadas del poder y de los ámbitos de decisión, en vez de compartir la mujer con el hombre el espacio público y privado.

En la encuesta realizada la segunda categoría más trascendental en la problemática de las mujeres estaba formada por problemas que se llamaron "culturales" con un porcentaje de 40,9\%, de los cuales Machismo fue mencionado con $27,8 \%$, y estereotipos, con $10,5 \%$.

El machismo, expresión derivada de la palabra "macho", se define como la actitud de prepotencia de los varones respecto a las mujeres.

Las respuestas concretas de las mujeres sobre Machismo fueron:

"El paradigma de la superioridad del hombre (machismo); nos creen con menos capacidades; machismo de los hombres en la parte cultural, 
laboral y educativa; aún se ve el machismo en algunos hogares; machismo imperante en la sociedad; machismo en algunas empresascírculo social; las mujeres hoy en día dejan que los hombres tomen las decisiones; todavía se ve la dependencia o mejor lo machista de la sociedad: los hombres toman las decisiones y las mujeres son dependientes y manipuladas por estos; el enfoque machista de la sociedad se expresa en las relaciones de pareja y laborales". (Fuente propia: encuestas a las mujeres). Estos son los estereotipos que se han mencionado:

"La tradición de nuestros padres; nos educaron para ser sumisas, hogareñas y buenas madres; limitaciones culturales para los desempeños laborales; la discriminación de la sociedad ante la capacidad de la mujer; discriminación por ser mujer, porque la creen sin capacidades de hacer cualquier actividad; hay quienes consideran que somos menos inteligentes que los varones; no nos dejan estudiar, no nos dejan trabajar, soy la que plancho, la que cocino; prohibir a las mujeres trabajar" (Fuente propia: encuestas a las mujeres).

Es de notar que no solo se evidencia el dominio de los hombres en la estructura social y la presión de estereotipos de la sociedad, sino que la propia conciencia de las mujeres se ve influenciada por estas posiciones sexistas y creencias patriarcales que dan a que 11,5\% de las mujeres tengan problemas en su actitud y en la creencia de en sus capacidades: "falta de actitud que nos lleve a asumir riesgos y retos que se presentan en todas las esferas de la sociedad; mentalidad conformista, dudas sobre su capacidad; inseguridad en sí mismas; falta de amor propio; la propia cultura de la mujer que se deja utilizar; falta de las ganas de salir adelante, de muchas mujeres; falta de conciencia de sí mismas para un objetivo común; falta de liderazgo con sentido propio" (Fuente propia: encuestas a las mujeres).

\section{Educación}

El $19,5 \%$ de las mujeres quindianas considera la falta de educación como un obstáculo para mejorar su vida. Para 5,5\% de las mujeres la mayor dificultad es tener el acceso al estudio por los altos costos. En el periodo comprendido entre el año 2001 y 2010, las mujeres recibieron el $57 \%$ de los títulos de Educación Superior, y los hombres, el restante $43 \%$. En total, 11172 títulos de pregrado (que incluyen la Educación Técnica, Tecnológica o Universitaria) y 2236 de posgrado (especialización, maestría o doctorado) han sido realizados por mujeres. En el nivel de licenciatura (pregrado) en el Quindío las mujeres recibieron $59,6 \%$ de títulos entre años 2001-2013 (SNIES, 2015) (Figura 4).

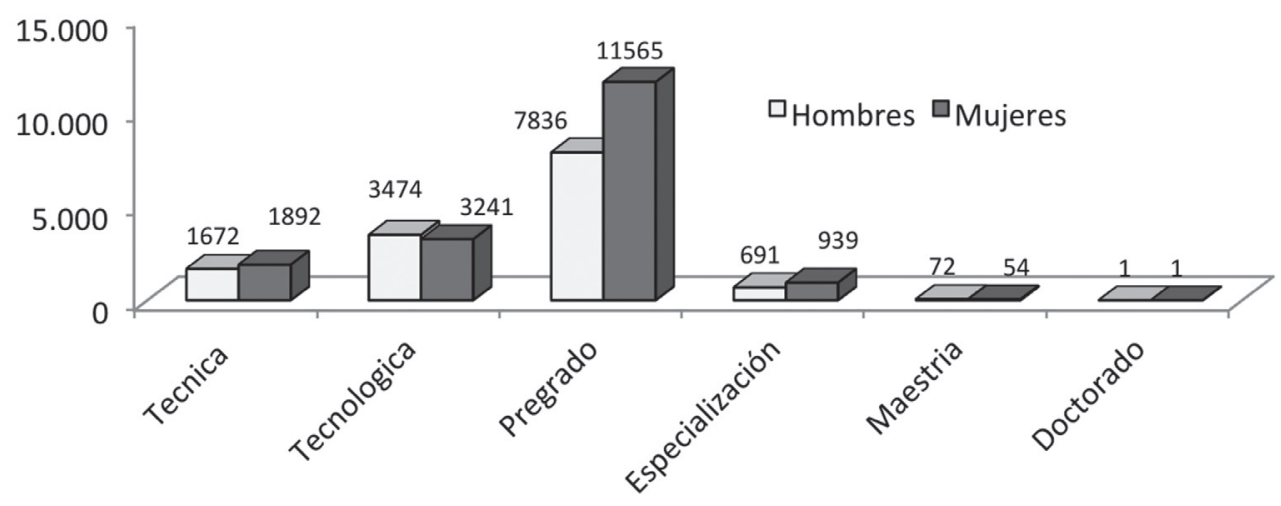

Figura 4. Títulos recibidos por nivel de estudios en los años 2001-2013, Quindío

Aunque el acceso a la educación se incrementó en todos los niveles y las mujeres son las que obtienen mayor cantidad de títulos universitarios y de especialización esto no se ve reflejado en la mejoría en cuanto a obtener los mismos salarios que los hombres.

En Colombia la igualdad de remuneración por un trabajo similar es de $59 \%$ para las 
mujeres en comparacion con los hombres, de acuerdo al Informe Global de Disparidad entre los Generos. Para la región se presenta una diferencia salarial de $7,1 \%$.

El ingreso promedio de un hombre que tenía su vinculación laboral en el Quindío para el año 2010 fue de $\$ 1.353 .915$ pesos. Para una mujer con pregrado solo fue $\$ 1.219 .138$ pesos, que es $90 \%$ del ingreso promedio de los hombres (Tabla 1).

En el nivel de posgrado esta diferencia aumenta: en promedio un hombre con posgrado obtiene 2.862.708 pesos, en tanto que una mujer con posgrado solo obtiene 2.251.755 pesos, que constituyen $78,7 \%$ del ingreso promedio del hombre (Tabla 1).

Tabla 1. Ingreso por nivel de estudios

\begin{tabular}{c|c|c|c|c}
\hline \multicolumn{2}{c|}{ NIVEL DE ESTUDIO } & Mujeres & Hombres & Ingreso (\%) \\
\hline \multirow{4}{*}{ Pregrado } & 2007 & 1.168 .707 & 1.334 .508 & 87,6 \\
\cline { 2 - 5 } & 2009 & 1.206 .824 & 1.353 .857 & 89,1 \\
\cline { 2 - 5 } & 2010 & 1.219 .138 & 1.353 .915 & 90,0 \\
\hline \multirow{3}{*}{ Posgrado } & 2007 & 2.055 .708 & 2.511 .684 & 81,8 \\
\cline { 2 - 5 } & 2009 & 2.244 .044 & 2.892 .312 & 77,6 \\
\cline { 2 - 5 } & 2010 & 2.251 .755 & 2.862 .708 & 78,7 \\
\hline
\end{tabular}

Para los años 2007, 2009 y 2010 disminuye la diferencia de ingresos para el nivel de pregrado, pero en el nivel de posgrado esta diferencia se aumenta (tabla 1). De acuerdo con Gómez (2010) en el Quindío para el año 2009 la diferencia de ingresos entre hombres y mujeres con nivel de posgrado fue de $22,4 \%$.

Entre los años 2001 y 2010, 299 mujeres obtuvieron títulos de maestría y 4 de doctorado, en tanto que para los hombres estas cifras son 327 para la maestría y 10 para el doctorado (tabla 1). Las mujeres se ven en mayores dificultades para acceder a maestrías o doctorados debido a dificultades económicas y dificultades relacionadas con los asuntos familiares: las mujeres deben conciliar su vida estudiantil con las obligaciones de cuidado de hogar e hijos; les falta de tiempo.

\section{Maltrato y violencia}

Un $21,6 \%$ de las mujeres afrontaron problemas de maltrato:

"Maltrato psicológico y violencia contra la mujer; abuso y maltrato físico y emocional; menosprecio y malos tratos; violencia por quienes nos creen inferiores y con menos capacidades; solo podemos hacer lo que el marido nos deja" (Fuente propia: encuestas a las mujeres).

El $14,3 \%$ no define bien la situación en la cual fue maltratada, pero $6,3 \%$ se refiere a maltrato intrafamiliar y otro $5,5 \%$ de las mujeres fueron abusadas sexualmente o estaban en las situaciones de acoso sexual.

En el Instituto Seccional de Salud del Quindío, ISSQ, en 2010 se reportaron 1831 denuncias a personas maltratadas y abusadas sexualmente. En 1311 casos las maltratadas son mujeres entre un año hasta los 44 años lo que equivale al 71,6 $\%$ de los casos presentados. Según el Centro de Atención a Víctimas de Agresión Sexual (CAlVAS), de la Fiscalía Seccional Quindío, entre el 2009 y el 2010 se han denunciado 379 casos por abuso sexual (Hincapié, 2012). Comparando estos datos con el porcentaje de las mujeres encuestadas se puede concluir que estos casos denunciados pueden representar apenas entre 7 y $12 \%$ del total de los casos de violencia intrafamiliar o abuso sexual. Sin embargo, la opinión de la defensora (Anónimo, 2012) es:

"Los casos de maltrato no han aumentado, lo que se ha incrementado es la denuncia, ahora ellas dan a conocer esos hechos sin temores". 


\section{Hogar, maternidad}

Los deberes de hogar, crianza de los hijos y responsabilidades en la familia son mencionados como problemas para $21,1 \%$ de las mujeres.

De las mujeres encuestadas $73 \%$ tiene hijos. Para $14,3 \%$ de las mujeres con hijos la maternidad crea un obstáculo en su vida laboral. En forma textual las mujeres encuestadas mencionan:

"Cuando decidimos ser madres en ocasiones encontramos limitantes; cuando hay que ir a trabajar y dejar los hijos con la empleada o solos; tener el deber de cuidar a los hijos con poco apoyo del esposo; la crianza de los hijos y ahora los nietos; estar dependiente del hogar y los hijos; la educación de los hijos está totalmente en manos de las madres, y los padres se han despreocupado; la responsabilidad para criar a los hijos o el madre-solterísimo $(5,3 \%$ son las madres solteras); la responsabilidad de criar los hijos nos la dejaron a las mujeres; la atención diaria a los hijos cuando están pequeños; tener demasiados hijos; tener mucha familia; amarrar a la mujer al hogar; crianza de los hijos".

El 2,0 \% de las mujeres afirma: "los esposos no quieren que sus parejas salgan adelante; que los esposos no las respetan". La carga laboral en el hogar, no remunerada, es considerada por el 8,3\% de las mujeres como un obstáculo en su vida. Además, la atención de los hijos y el trabajo generan una sobrecarga de responsabilidades concentrada en las mujeres. Por tal razón, al $0.8 \%$ de las mujeres les falta tiempo para cumplir con sus sueños.

\section{Conclusiones}

En el departamento del Quindío se presenta una situación bastante complicada con el empleo femenino, con las dificultades de tipo económico que afronta $57,9 \%$ de las mujeres del segundo destino turístico del país. La falta de empresas productivas y proyectos que generen empleo para las mujeres llevan a que las cifras de desempleo femenino estén mucho más altas para las mujeres que para los hombres. Las obligaciones de cuidado de hijos y ancianos no les permiten a muchas mujeres tener un empleo formal. Se encontró que de las mujeres encuestadas mayores de 65 años, ninguna recibía una pensión, y la subsistencia de ellas es únicamente un asunto de generosidad de sus familiares.

Aunque las mujeres obtienen más títulos profesionales que los hombres, sigue presentándose una tasa más baja de ocupación para las mujeres que para los hombres; las más vulnerables son las mujeres mayores de 45 años y mujeres con hijos. Persiste el desnivel de los ingresos más marcado para las mujeres con niveles de educación de posgrado que de pregrado.

Finalmente se necesitan cambios ideológicos en lo que tiene que ver con la posición de la mujer en la sociedad. Los cambios culturales se pueden hacer por medio de los métodos de comunicación masiva: televisión y radio, campañas publicitarias, mostrando ejemplos de mujeres exitosas, profesionales, empresarias; asimismo, dando a conocer las dificultades de las mujeres de diferentes países y sus logros en la vida social y pública.

Educación y la pobreza en Colombia tienen su regionalización: en las ciudades grandes hay mayor desarrollo de capital humano que en las regiones. De allí la falta de oportunidades de capacitación para las mujeres por escasa variedad y muy pocas entidades que ofrecen los estudios de más alto nivel como las maestrías y doctorados en la región del Quindío. Debería considerarse la posibilidad de hacer más sedes de los principales universidades en las ciudades pequeñas, para que las mujeres casadas y con hijos pueden acceder a los estudios de postgrados, así como la implementación de mayor cantidad de carreras a distancia.

\section{Agradecimientos}

Los autores agradecen a la Universidad del Quindío por el apoyo dado que suscitó la elaboración de este artículo.

J. D. Mosquera-Artamonov agradece a la CONACYT por la beca de postgrado. 


\section{Referencias bibliográficas}

- Hausamann, R.; Tyson L. y Zadich S. (2010). World Economic Forum. Global Gender Gap. Switzerland: Report. World Economic Forum

- Solano D. S. P. y Flórez B. R. (2013). Política y trabajo. Debates sobre las normas jurídicas y las relaciones laborales de servidumbre en el Bolívar Grande (Colombia) en el siglo XIX. Revista de Estudios Sociales. (45). P 16-28.

- Monge, R. V. (2006). Guía de indicadores para la evaluación del impacto de género de las políticas públicas. Açores. Madeira: Feder.

- Gaviria U. A. y Henao V. M. L (2001). Comportamiento del desempleo en los últimos años y estrategias para enfrentarlo. En: Revista Coyuntura Social (24)23-38. Fedesarrollo. Bogotá. Mayo.

- Departamento Administrativo Nacional de Estadística DANE (2005). Boletín censo general. Perfil Quindío. Recuperado de http://www. dane.gov.co/files/censo2005/PERFIL_PDF_ CG2005/63000T7T000.PDF.

- Gómez, O. (2010). Observatorio del mercado laboral y el empleo de Armenia, Alcaldía de Armenia. Recuperado de: http://www.almamater. edu.co/sitio/Archivos/Documentos/Documentos/00000118.pdf.

- Martínez Bencardino, C. (2005). Estadística y muestreo. Decima Segunda Edición. Ecoe Ediciones. Bogotá. Colombia.

- Crónica del Quindío, (2010). Diplomado reinventando la ciudadanía de las Mujeres. Consejo Municipal de Mujeres de Calarcá. 22/09/2010. Recuperado de: http://www.cronicadelquindio. com/noticia-completa-titulo-diplomado_gratuito_reinventando_la_ciudadana_de_las_mujeres-seccion-la_regin-nota-19015.

- Departamento Administrativo Nacional de Estadística DANE. (2011). Boletín de prensa. Mercado laboral por sexo gran encuesta integrada de hogares. Recuperado de http://www.dane. gov.co/files/investigaciones/boletines/ech/juventud/boletin_trim_ene_mar13.pdf.

- Amnistía Internacional. (2004). Colombia Cuerpos marcados, crímenes silenciados. Violencia sexual contra las mujeres en el marco del conflicto armado. Londres: Artes Gráficas ENCO, S. L.

- Portafolio.co (2010). En Colombia las mujeres sufren discriminación salarial, dice estudio. Recuperado de: http://www.portafolio.co/archivo/ documento/CMS-7796903

- El Colombiano. (2012). Presidente Santos firmó acuerdo en lucha contra la discriminación de la mujer. Histórico. Recuperado de: http:// www.elcolombiano.com/discriminacion_contra_ la_mujer_presidente_santos_firmo_acuerdoDB̈EC_172643.

- Rodríguez P. G. y Flórez V. G. (2007). Migraciones internacionales y explotación sexual comercial. Revista Interdisciplinar da Mobilidade Humana. 2007. Vol. 29. Pp. 87-108.

- Cano, C. y Orozco, M. (2011). Diferenciales Salariales en el Área Metropolitana de Medellín, a través de regresiones por cuantiles en el período 2002-2006. En: Ecos de Economía. 2011, 15(32), 59-97.

- Daza-Caicedo, S y Pérez-Bustos, T. (2008). Contando mujeres. Una reflexión sobre los indicadores de género y ciencia en Colombia. Revista de Antropología y sociología Virajes. (10)29-51

- Buquet Corleto, A, Cooper, J. y Rodríguez, H. (2010). Sistema de indicadores para la equidad de género en instituciones de educación superior. México: UNAM. Recuperado de: http:// www.pueg.unam.mx/images/equidad/investigacion/eisistindi.pdf

- Gutiérrez, A. (2010). Educación y mercado laboral en Santander: una mirada en perspectiva de género. Revista Lebret. 2010, 2(2), 47-69.

- Del Castillo, M.; Roth D. A. N.; Patiño W. C. I.; Higuiera, R. R. y Chacón N. B. O. A. (2008). La implementación de la política pública de salud sexual y reproductiva (SSR) en el Eje Cafetero colombiano: el caso del embarazo adolescente. Revista Latinoamericana de Ciencias Sociales, Niñez y Juventud. 2008. 6(1) pp. 217-255.

- SNIES. (2015). Sistema Nacional de Información de la Educación Superior del Ministerio de Educación Nacional. Recuperado de: http:// bi.mineducacion.gov.co:8080/o3web/jdesktop.jsp

- Hurtado D. Y. (2012). 1.311 casos de violencia contra las mujeres en 2010 en Quindío. Crónica del Quindío.Com. Recuperado de: http://www. cronicadelquindio.com/noticia-completa-titulo-1_311_casos_de_violencia_contra_las_mujeres_en_2010_en_quindio-seccion-Regionalnota-26605.htm

- Hincapié, L. (2012). Aumentan denuncias por abuso sexual infantil en Quindío. Temas del día. Recuperado de: http://www.eltiempo.com/ archivo/documento/CMS-13640706.

- Anónimo. (2012). 1.343 casos de maltrato a mujeres en Quindío. Crónica del Quindío. Recuperado de http://www.cronicadelquindio. com/noticia-completa-titulo-1_343_casos_de_ maltrato_a_mujeres_en_quindio-seccion--nota-39379.htm 